\title{
ON CERTAIN INTEGRAL EQUATIONS OF CONVOLU- TION TYPE WITH BESSEL-FUNCTION KERNELS
}

\author{
by R. P. SRIVASTAV
}

(Received 2nd August 1965)

1. In this paper we first obtain elementary solutions of the integral equations

$$
\int_{a}^{x} \psi(\rho)\left(x^{2}-\rho^{2}\right)^{\frac{1}{2} \beta} J_{\beta}\left\{\sqrt{\kappa\left(x^{2}-\rho^{2}\right)}\right\} d \rho=m(x), \quad a \leqq x \leqq b
$$

and

$$
\int_{x}^{b} \psi(\rho)\left(\rho^{2}-x^{2}\right)^{\frac{1}{2} \beta} J_{\beta}\left\{\sqrt{\kappa\left(\rho^{2}-x^{2}\right)}\right\} d \rho=m(x), \quad a \leqq x \leqq b .
$$

Using these solutions we then define operators of fractional integration. These operators may be regarded as a generalisation of the operators of fractional integration introduced by Sneddon (1) as a modification of Erdélyi-Kober operators. In fact Erdélyi-Kober-Sneddon operators may be obtained by multiplying both sides of the equations by $\kappa^{-\frac{1}{2}}$ and considering the limiting case $\kappa \rightarrow 0$. We employ these operators to find a generalisation of the Mellin transform.

The integral equations (1.1) and (1.2) appear in Burlak's (2) investigation of dual integral equations

$$
\begin{aligned}
& \int_{\kappa}^{\infty} u^{-v-\mu}\left(u^{2}-\kappa^{2}\right)^{\alpha} \psi(u) J_{\mu}(x u) d u=f(x), \quad 0 \leqq x<1, \\
& \int_{0}^{\infty} \psi(u) J_{v}(x u) d u \quad=g(x), \quad x>1 .
\end{aligned}
$$

The integral equations (1.1) and (1.2) are of convolution type and as is usual for such equations Burlak uses Laplace transforms for their solution. The solution obtained is however valid only for $-1<\beta<0$ and the formal solution found using Laplace transforms yields wrong answers for $\beta \geqq 0$. For example, consider the integral equation

$$
\int_{0}^{x} \psi(\rho) J_{0}\left\{\sqrt{\kappa\left(x^{2}-\rho^{2}\right)}\right\} d \rho=\sin x, \quad 0 \leqq x<\infty .
$$

The solution using Burlak's results is

$$
\psi(x)=\sqrt{\kappa} \frac{d}{d x} \int_{0}^{x} \rho \sin \rho\left(x^{2}-\rho^{2}\right)^{-\frac{1}{2}} I_{1}\left\{\sqrt{\kappa\left(x^{2}-\rho^{2}\right)}\right\} d \rho .
$$


Integrating by parts we find that

$$
\psi(x)=\frac{d}{d x}\left[\int_{0}^{x} \cos t I_{0}\left\{\sqrt{\kappa\left(x^{2}-t^{2}\right)}\right\} d t-\sin x\right]
$$

and since

$$
\int_{0}^{x} \cos t I_{0}\left\{\sqrt{\kappa\left(x^{2}-t^{2}\right)}\right\} d t=\frac{1}{\sqrt{(1-\kappa)}} \sin (x \sqrt{(}(1-\kappa)),
$$

it easily follows that

$$
\psi(x)=\sqrt{\kappa}[\cos (x \sqrt{ }(1-\kappa))-\cos x] .
$$

However

$$
\begin{aligned}
\sqrt{\kappa} \int_{0}^{x}\{\cos (\rho \sqrt{ }(1-\kappa))-\cos \rho\} & \left.J_{0}\left\{\sqrt{\kappa\left(x^{2}-\rho^{2}\right.}\right)\right\} d \rho \\
& =\sqrt{\kappa}[\sin x-\sqrt{ }(1+\kappa) \sin (x \sqrt{ }(1+\kappa))]
\end{aligned}
$$

and therefore $\psi(x)$ given by (1.6) is not a solution of the equation (1.5).

2. The solution of the equations (1.1) and (1.2) given here depends on Sonine's integral (3)

$$
\begin{aligned}
& \int_{0}^{\pi / 2}(\cos \theta)^{\mu+1}(\sin \theta)^{\nu+1} J_{\mu}(a \cos \theta) J_{v}(b \sin \theta) d \theta \\
& \quad=a^{\mu} b^{\nu}\left(a^{2}+b^{2}\right)^{-\frac{1}{2}(\mu+v+1)} J_{\mu+\nu+1}\left(\sqrt{ }\left(a^{2}+b^{2}\right)\right) ; \quad \mu>-1, v>-1 .
\end{aligned}
$$

We consider the cases (i) $-1<\beta<0$, (ii) $\beta=0$ and (iii) $\beta>0$ separately. We give the detailed solution of the equation (1.1) and only mention the final result for the equation (1.2).

For $-1<\beta<0$, to solve the integral equation (1.1), we consider the integral

$$
F(t)=\int_{a}^{t} x m(x)\left(t^{2}-x^{2}\right)^{-\frac{1}{2}(\beta+1)} J_{-(1+\beta)}\left\{\sqrt{-\kappa\left(t^{2}-x^{2}\right)}\right\} d x
$$

If we now put the value of $m(x)$ in terms of $\psi$ from the equation (1.1) we find on changing the order of integration that

$$
\begin{aligned}
F(t)=\int_{a}^{t} \psi(\rho) & \left(\int_{\rho}^{t}\left(t^{2}-x^{2}\right)^{-\frac{1}{2}(1+\beta)}\left(x^{2}-\rho^{2}\right)^{\frac{1}{2} \beta} x\right. \\
& \left.\times J_{\beta}\left\{\sqrt{\kappa\left(x^{2}-\rho^{2}\right)}\right\} J_{-(1+\beta)}\left\{\sqrt{-\kappa\left(t^{2}-x^{2}\right)}\right\} d x\right) d \rho .
\end{aligned}
$$

By a trivial change of the variable the equation (2.3) can be put in the form

$$
\begin{gathered}
F(t)=\int_{a}^{t} \psi(\rho)\left(t^{2}-\rho^{2}\right)^{\frac{1}{2}} \int_{0}^{\pi / 2}(\sin \theta)^{\beta+1}(\cos \theta)^{-\beta} \\
\left.\left.\quad J_{\beta}\left(\sqrt{\kappa\left(t^{2}-\rho^{2}\right.}\right) \sin \theta\right) J_{-(1+\beta)}\left(\sqrt{-\kappa\left(t^{2}-\rho^{2}\right.}\right) \cos \theta\right) d \theta d \rho .
\end{gathered}
$$


Using the relation (2.1) we now get

from which it immediately follows that

$$
F(t)=\kappa^{\frac{1}{2} \beta}(-\kappa)^{-\frac{1}{2}(1+\beta)} \int_{a}^{t} \psi(\rho) d \rho,
$$

$$
\psi(t)=\kappa^{\frac{1}{2}} \frac{d}{d t} \int_{a}^{t} x m(x)\left(t^{2}-x^{2}\right)^{-\frac{1}{2}(\beta+1)} I_{-(1+\beta)}\left(\sqrt{\kappa\left(t^{2}-x^{2}\right)}\right) d x .
$$

The above solution is in agreement with that found by Burlak.

If $\beta=0$, we proceed by taking into account the integral

$$
F(t)=\sqrt{\kappa} \int_{a}^{t} \frac{d}{d \rho} m(\rho) I_{0}\left\{\sqrt{\kappa\left(t^{2}-\rho^{2}\right)}\right\} d \rho .
$$

Simple integration by parts leads to the equation

$$
F(t)=\sqrt{\kappa m}(t)+\kappa \int_{a}^{t} \rho m(\rho)\left(t^{2}-\rho^{2}\right)^{-\frac{1}{2}} I_{1}\left\{\sqrt{\kappa\left(t^{2}-\rho^{2}\right)}\right\} d \rho .
$$

If we replace $m(\rho)$ by its expression in (1.1) in terms of $\psi$, we obtain the equation

$$
F(t)=\sqrt{\kappa} m(t)+\kappa \int_{a}^{t} \psi(y) d y \int_{y}^{t} \frac{\left.\left.\rho J_{0}\left(\sqrt{\kappa\left(\rho^{2}-y^{2}\right.}\right)\right) I_{1}\left(\sqrt{\kappa\left(t^{2}-\rho^{2}\right.}\right)\right)}{\sqrt{\left(t^{2}-\rho^{2}\right)}} d \rho
$$

which leads to the equation

$$
F(t)=\sqrt{\kappa} m(t)+\sqrt{\kappa} \int_{a}^{t} \psi(y)\left\{1-J_{0}\left(\sqrt{\kappa\left(t^{2}-y^{2}\right)}\right) d y,\right.
$$

finally giving the solution as

$$
\left.\psi(\rho)=\frac{d}{d \rho} \int_{a}^{\rho} \frac{d}{d x} m(x) I_{0}\left(\sqrt{\kappa\left(\rho^{2}-x^{2}\right.}\right)\right) d x .
$$

In case $\beta \geqq 0$, we have to perform successive reduction by introducing

$$
\begin{aligned}
& \psi_{1}(\rho)=\kappa^{\frac{1}{2}} \int_{a}^{\rho} \psi(t) d t \\
& \cdot \cdot \cdot \cdot \cdot \cdot \cdot \\
& \psi_{n}(\rho)=\kappa^{\frac{1}{2}} \int_{a}^{\rho} t \psi_{n-1}(t) d t
\end{aligned}
$$

and integrating by parts till $-1<\beta^{\prime} \leqq 0$, where $\beta^{\prime}$ is the order of Bessel Function involved in the integral equation. For example, the equation (1.1) is equivalent to the equation

$$
\left.\int_{a}^{x} \rho \psi_{1}(\rho)\left(x^{2}-\rho^{2}\right)^{\frac{1}{2}(\beta-1)} J_{\beta-1}\left(\sqrt{\kappa\left(x^{2}-\rho^{2}\right.}\right)\right) d \rho=m(x),
$$

which can in turn be reduced to the equation

$$
\int_{a}^{x} \rho \psi_{2}(\rho)\left(x^{2}-\rho^{2}\right)^{\frac{1}{2}(\beta-2)} J_{\beta-2}\left(\sqrt{\kappa\left(x^{2}-\rho^{2}\right)}\right) d \rho=m(x) .
$$


As an illustration consider the equation

If we put

$$
\int_{0}^{t} \sin \left(\sqrt{\kappa\left(t^{2}-x^{2}\right)}\right) \psi(x) d x=t J_{1}(t), \quad 0 \leqq t<\infty .
$$

$$
\psi_{1}(x)=\kappa^{\frac{1}{2}} \int_{0}^{x} \psi(u) d u,
$$

then we find that $\psi_{1}(x)$ satisfies the equation

$$
\int_{0}^{t} x \psi_{1}(x) \frac{\cos \left(\sqrt{\kappa\left(t^{2}-x^{2}\right)}\right)}{\sqrt{\left(t^{2}-x^{2}\right)}} d x=t J_{1}(t)
$$

which can be written in the alternative form

$$
\sqrt{\frac{\pi}{2}} \kappa^{\frac{1}{4}} \int_{0}^{t} x \psi_{1}(x)\left(t^{2}-x^{2}\right)^{-\frac{1}{4}} J_{-\frac{1}{2}}\left(\sqrt{\kappa\left(t^{2}-x^{2}\right)}\right) d x=t J_{1}(t)
$$

and therefore has the solution

i.e.

$$
x \psi_{1}(x)=\sqrt{\frac{2}{\pi}} \kappa^{\frac{1}{4}} \frac{d}{d x} \int_{0}^{x} t^{2} J_{1}(t)\left(x^{2}-t^{2}\right)^{-\frac{1}{d}} I_{-\frac{1}{2}}\left(\sqrt{\kappa\left(x^{2}-t^{2}\right)}\right) d t
$$

$$
x \psi_{1}(x)=\frac{2}{\pi} \frac{d}{d x} \int_{0}^{x} \frac{\left.t^{2} J_{1}(t) \cosh \left(\sqrt{\kappa\left(x^{2}-t^{2}\right.}\right)\right) d t}{\sqrt{\left(x^{2}-t^{2}\right)}} .
$$

The integral in the equation (2.19) can be evaluated using convolution theorem for Laplace transforms. It is easily shown that

$$
\psi(x)=\frac{2}{\pi \sqrt{\kappa}} \cos (x \sqrt{(}(1-\kappa))
$$

Using the relation (37), p. 27 of (4) we can verify that $\psi(x)$ satisfies the equation (2.15).

In a similar manner we can show that for $-1<\beta<0$, the solution of the equation (1.2) is given by the relation

$$
\psi(\rho)=\sqrt{\kappa} \frac{d}{d \rho} \int_{\rho}^{b} x m(x)\left(x^{2}-\rho^{2}\right)^{-\frac{1}{2}(\beta+1)} I_{-(1+\beta)}\left\{\sqrt{\kappa\left(x^{2}-\rho^{2}\right)}\right\} d x .
$$

The equation

has the solution

$$
\int_{x}^{b} \psi(\rho) J_{0}\left\{\sqrt{\kappa\left(\rho^{2}-x^{2}\right)}\right\} d \rho=m(x)
$$

$$
\psi(x)=\frac{d}{d x} \int_{x}^{\infty} \frac{d}{d t} m(t) I_{0}\left(\sqrt{\kappa\left(t^{2}-x^{2}\right)}\right) d t .
$$

For $\beta>0$ the process of successive reduction is employed to give an integral equation such that the Bessel function involved is of order $v$, where $-1<v \leqq 0$. 
3. Define for $v>0$,

$\Im_{\eta, v, \kappa}(f)=2^{v} \kappa^{\frac{1}{2}-\frac{t}{v} t^{-2 \eta-2 v}} \int_{0}^{t} x^{2 \eta+1}\left(t^{2}-x^{2}\right)^{t v-\frac{1}{2}} J_{v-1}\left(\sqrt{\kappa\left(t^{2}-x^{2}\right)}\right) f(x) d x$

$\Re_{\eta, v, \kappa}(f)=2^{v} \kappa^{\frac{1}{2}-\frac{1}{2} v} t^{2 \eta} \int_{t}^{\infty} x^{1-2 \eta-2 v}\left(x^{2}-t^{2}\right)^{\frac{1}{2 v} v-\frac{1}{2}} J_{v-1}\left(\sqrt{\kappa\left(x^{2}-t^{2}\right)}\right) f(x) d x$.

Using Sonine's formula it is easy to verify that

and that

$$
\mathfrak{I}_{\eta+v, \mu, l-\kappa} \mathfrak{J}_{\eta, v, \kappa}=\mathfrak{I}_{\eta, v+\mu, l}
$$

We observe that

$$
\boldsymbol{R}_{\eta, v, \kappa} \boldsymbol{R}_{\eta+v, \mu, l-\kappa}=\boldsymbol{\Omega}_{\eta, \mu+v, l}
$$

$$
\begin{aligned}
& \mathfrak{I}_{\eta, v, 0}=I_{\eta, v}, \\
& \Re_{\eta, v, 0}=K_{\eta, v},
\end{aligned}
$$

where $I_{\eta, v}$ and $K_{\eta, v}$ are Erdélyi-Kober-Sneddon operators of fractional integration. If we put $\kappa=l$ and $\mu=0$ formally in the equations (3.3) and (3.4) we find that $\mathfrak{I}_{\eta, 0,0}$ and $\boldsymbol{\Omega}_{\eta, 0,0}$ should behave as identity operators and that the inverses should be given by the relations

$$
\begin{aligned}
& \left(\mathfrak{I}_{\eta, v, \kappa}\right)^{-1}=\mathfrak{I}_{\eta+v,-v,-\kappa}, \\
& \left(\Re_{\eta, v, \kappa}\right)^{-1}=\mathfrak{K}_{\eta+v,-v,-\kappa} .
\end{aligned}
$$

We therefore define for $v=0$,

$$
\begin{aligned}
& \mathfrak{I}_{\eta, 0, \kappa}=t^{-2 \eta} \int_{0}^{t} \frac{d}{d x}\left(x^{2 \eta} f(x)\right) J_{0}\left(\sqrt{\kappa\left(t^{2}-x^{2}\right)}\right) d x, \\
& \Re_{\eta, 0, \kappa}=t^{2 \eta} \int_{t}^{\infty} \frac{d}{d x}\left(x^{-2 \eta} f(x)\right) J_{0}\left(\sqrt{\kappa\left(x^{2}-t^{2}\right)}\right) d x
\end{aligned}
$$

and for $v<0, \mathfrak{I}_{\eta, v, \kappa}(f)$ and $\Omega_{\eta, v, \kappa}(f)$ are defined to be respectively the solutions of the integral equations

and

$$
\mathfrak{I}_{n+v,-v,-\kappa} g=f
$$

$$
\Re_{\eta+v,-v,-\kappa} g=f \text {. }
$$

\section{A generalised Mellin transform}

Now we give a formal generalisation of the well-known Mellin transform defined by the relation

with the inversion formula

$$
\mathfrak{M}(\psi) \equiv \Psi(s)=\int_{0}^{\infty} \psi(\rho) \rho^{s-1} d \rho
$$

$$
\mathfrak{M}^{-1}(\Psi) \equiv \psi(\rho)=\frac{1}{2 \pi i} \int_{c-i \infty}^{c+i \infty} \Psi(s) \rho^{-s} d s
$$

valid under fairly general conditions. The present generalisation is heuristic and no attempt is made to determine the precise conditions under which the inversion formula holds. 
We define a generalised Mellin transform of a function $\psi(\rho)$ by means of the relation

$$
\Psi(s)=\int_{0}^{\infty} \rho^{\beta+\frac{1}{2} s} I_{\beta+\frac{1}{2} s}(\kappa \rho) \psi(\rho) d \rho, \quad-1<\beta<0 .
$$

Multiplying both the sides of the above equation by $2^{\frac{1}{2} s-1} \Gamma\left(\frac{1}{2} s\right) \kappa^{-\frac{1}{2} s}$ and considering the inverse Mellin transform with respect to $s$, we get using the relation ((4), (13), p. 329), the equation

$$
\mathfrak{M}^{-1}\left\{\Psi(s) 2^{\frac{1}{2} s-1} \Gamma\left(\frac{1}{2} s\right) \kappa^{-\frac{1}{2} s}\right\}=\int_{x}^{\infty}\left(\rho^{2}-x^{2}\right)^{\frac{1}{2}} I_{\beta}\left(\kappa \sqrt{ }\left(\rho^{2}-x^{2}\right)\right) \psi(\rho) d \rho .
$$

The equation (4.4) is of the form (1.2) and its solution is given by the equation (2.21). Hence

$$
\begin{aligned}
\psi(\rho)=-\kappa \frac{d}{d \rho} \frac{1}{2 \pi i} \int_{c-i \infty}^{c+i \infty} \Psi(s) 2^{\frac{1}{2} s-1} \Gamma\left(\frac{1}{2} s\right) \kappa^{-\frac{1}{2} s} \\
\quad \times \int_{\rho}^{\infty} x^{1-s}\left(x^{2}-\rho^{2}\right)^{-\frac{1}{2}(\beta+1)} J_{-(1+\beta)}\left(\kappa \sqrt{ }\left(x^{2}-\rho^{2}\right) d x d s .\right.
\end{aligned}
$$

By a change of variable and taking into account the relation $((5),(20)$, p. 24) we can easily show that

$$
\begin{aligned}
\int_{\rho}^{\infty} x^{1-s}\left(x^{2}-\rho^{2}\right)^{-\frac{1}{2}(\beta+1)} J_{-(\beta+1)}\left(\kappa \sqrt{ }\left(x^{2}-\rho^{2}\right)\right) d x \\
= \\
=\frac{\rho^{-\beta-\frac{1}{2} s} \kappa^{\frac{1}{2} s-1} K_{-\beta-\frac{1}{2} s}(\kappa \rho)}{2^{\frac{1}{2} s-1} \Gamma\left(\frac{1}{2} s\right)} .
\end{aligned}
$$

It therefore follows that

$$
\psi(\rho)=\frac{\kappa}{2 \pi i} \int_{c-i \infty}^{c+i \infty} \rho^{-\beta-\frac{1}{2} s} K_{-\beta-\frac{1}{2} s-1}(\kappa \rho) \Psi(s) d s
$$

\section{REFERENCES}

(1) I. N. SNEDDON, Fractional Integration and Dual Integral Equations, North Carolina State College Applied Mathematics Research Group, Report PSR-6 (1962).

(2) J. BURLAK, A pair of dual integral equations occurring in diffraction theory, Proc. Edinburgh Math. Soc. 13 (1962), 179-187.

(3) A. ERDÉLyi et al., Higher Transcendental Functions, Vol. 2 (McGrawHill, New York, 1953).

(4) A. ERDÉLYi et al., Tables of Integral Transforms, Vol. 1 (McGraw-Hill, New York, 1954).

(5) A. ERDÉLyI et al., Tables of Integral Transforms, Vol. 2 (McGraw-Hill, New York, 1954).

\section{Department of Mathematics \\ DUKE UNIVERSITY \\ DURHAM, N.C.}

